\title{
Providing multi-user in NCL with userAgent and UserProfile
}

\author{
Fábio Barreto \\ MídiaCom Lab - UFF \\ UNILASALLE-RJ \\ fbarreto@midiacom.uff.br
}

\author{
Eyre Brasil B. Montevecchi \\ MídiaCom Lab - UFF \\ eyrebrasil@midiacom.uff.br
}

\author{
Raphael Abreu \\ MídiaCom Lab - UFF \\ raphael.abreu@midiacom.uff.br
}

\author{
Joel A. F. dos Santos \\ CEFET/RJ \\ jsantos@eic.cefet-rj.br
}

\author{
Débora C. Muchaluat-Saade \\ MídiaCom Lab - UFF \\ debora@midiacom.uff.br
}

\begin{abstract}
This proposal consists of adding a new language module named Users to provide multi-user support in NCL, proposing three new NCL elements: < userBase >, < userAgent > and <userProfile > . userBase is used for declaring user and their profiles. userAgent identifies a user and userProfile represents a description of the user's profile. Each userAgent is associated with only one userProfile, and together, they allow representing a unique user. The user description with its specific characteristics can be loaded from a profile specified with the Resource Description Framework (RDF) standard, for example.
\end{abstract}

\section{KEYWORDS}

Multi-user, NCL, IoT, Authoring

\section{BACKGROUND}

A promessa de aplicações multiusuário tem levado a diversas evoluções na forma de desenvolvimento de aplicações multimídia. Em ambientes interativos, a adição de múltiplos usuários implica a necessidade de identificação de tais usuários para poderem interagir com o sistema. Para permitir autoria dessas aplicações, a linguagem deve permitir que o autor identifique cada usuário individualmente. Porém, identificar um usuário não é fácil do ponto de vista de autoria. Visto que o código gerado seria altamente acoplado ao identificador do usuário do sistema. Outro problema é que devem ser informados especificamente quais usuários e quantos usuários. Por outro lado, perfis de usuários podem ser definidos mais facilmente pelo autor, por exemplo.

\section{PROPOSAL}

A linguagem NCL ainda não possui suporte a multi-usuário, apesar de propostas anteriores sugerirem tal facilidade [1]. Para que isso seja possível, esta proposta consiste na adição de um novo módulo de linguagem chamado Users, que propõe três novos elementos NCL: <userBase>, que é usado para declarar usuários e seus perfis, <userAgent>, que identifica um usuário que pode interagir com a aplicação NCL, e <userProfile>, que representa a descrição do perfil do usuário. Essa descrição do usuário com suas características específicas pode ser carregada de um perfil especificado com padrão Resource Description Framework (RDF), por exemplo, contendo desde

In: Future of Interactive Television Workshop (V WTVDI), Rio de Janeiro, Brasil. Anais Estendidos do Simpósio Brasileiro de Sistemas Multimídia e Web (WebMedia). Porto Alegre: Sociedade Brasileira de Computação, 2019.

ISSN 2596-1683 preferências de tipos de mídia até limiares de percepção como volume de som, luminosidade, etc.

Cada userAgent está associado a somente um userProfile e juntos, permitem representar cada usuário individualmente. A Listagem 1 mostra um exemplo de definição de dois perfis de dois usuários, sendo possível inclusive referenciar um arquivo RDF com suas características especificadas.

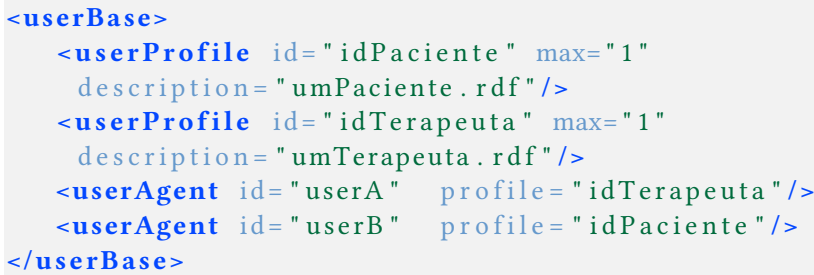

Listagem 1: Definição de características de mais de um usuário de perfil diferente participante da aplicação

\section{USE CASE(S)}

O caso de uso que ilustra a utilização dos novos elementos < userBase $>$, < userAgent $>$ e $<$ userProfile > é uma aplicação com objetivo de ajudar na realização de terapias de Integração Sensorial em indivíduos com TEA (Transtorno do Espectro Autista). Essa consiste em orquestrar um ambiente imersivo onde estaria inserido o indivíduo (João) e a sua terapeuta (Marina). São proporcionadas várias experiências sensoriais controladas pelo terapeuta. A Listagem 2 contém a especificação de parte do funcionamento desse ambiente, onde o vídeo (trafficVideo) pode ser interrompido quando o paciente demonstrar insatisfação através de seu semblante.

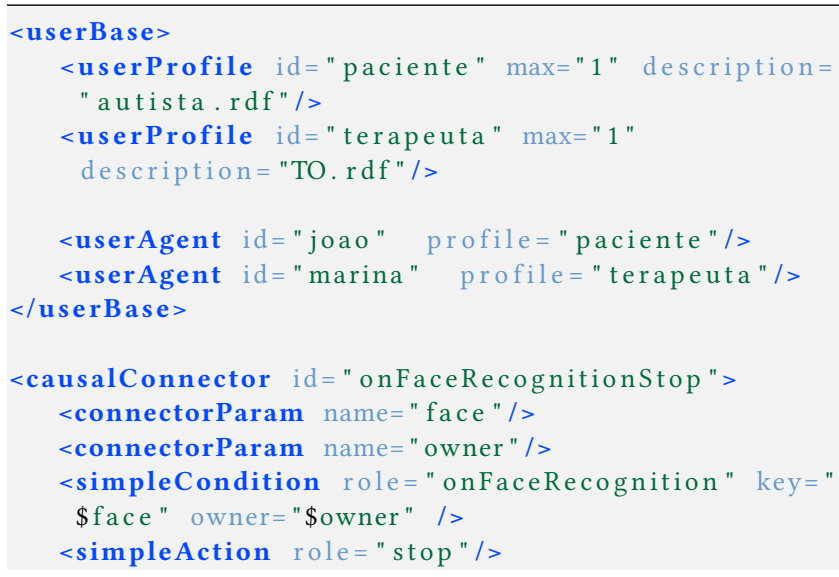




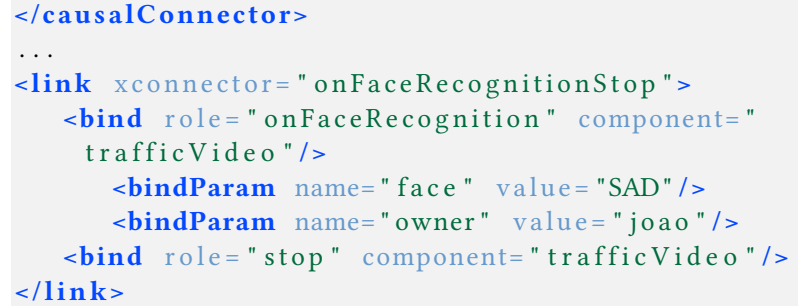

Listagem 2: Especificação de exemplo para uso de $<$ userAgent $>$ e $<$ userProfile $>$

\section{ACKNOWLEDGMENTS}

Os autores gostariam de agradecer a CAPES, CNPq e FAPERJ pelo apoio financeiro parcial deste trabalho.

\section{REFERENCES}

[1] Álan Lívio Vasconcelos Guedes, Roberto Gerson de Albuquerque Azevedo, and Simone Diniz Junqueira Barbosa. 2017. Extending multimedia languages to support multimodal user interactions. Multimedia Tools and Applications 76, $4(01 \mathrm{Feb}$ 2017), 5691-5720. https://doi.org/10.1007/s11042-016-3846-8 